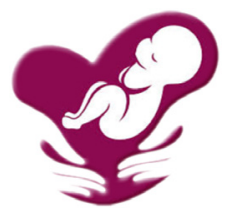

Published by DiscoverSys

\title{
The role of circular Ribonucleic Acid in preeclampsia: a literature review
}

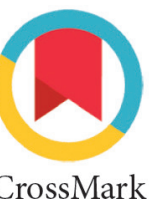

\author{
William Alexander Setiawan ${ }^{1 *}$, A.A Gde Marvy Khrisna Pranamartha ${ }^{1}$
}

'Department of Obstetrics and Gynecology, Faculty of Medicine, Universitas Udayana/Sanglah General Hospital, Denpasar, Bali, Indonesia
*Corresponding to:

William Alexander Setiawan.

Department of Obstetrics and Gynecology, Faculty of Medicine, Universitas Udayana/Sanglah General Hospital, Denpasar, Bali, Indonesia;

William.alexanders11@gmail.com

Received: 2020-06-27 Accepted: 2020-08-12 Published: 2020-08-26
The circular RNAs (circRNAs) are part of the non-coding RNAs (ncRNA) family. The circRNAs is an ncRNA that is known to have a closedcircular structure linked by covalent-bond. It is known that circular RNA plays an essential role in normal biological function as well as in certain diseases processes. Researchers have identified several circRNAs which can be used as a biomarker of several diseases, such as neurodegenerative disorders, tumors, cardiovascular diseases, and various tissue injuries. ${ }^{1-3}$ Numerous studies have shown that various ncRNAs, including circRNAs involved in the development and progress of pregnancy-related illnesses. This review will highlight the biological basis of circRNAs and its relation to the pathogenesis of preeclampsia.

\section{WHAT IS CIRCRNA?}

As mentioned before, the RNAs with a circular structure are referred to as circRNAs. ${ }^{4}$ The circRNAs do not have a polarity end (the $5^{\prime}$ or $3^{\prime}$ ) nor the polyadenylate end, thus they spared from degradation by RNases and thus relatively stable. Previously, the term "circRNA" usually refers to the circRNAs that are derived from exons, due to consideration that the proportion of other types circRNAs was far smaller. ${ }^{5}$ The biogenesis process involves back splicing of exon and/or intron resulted in the inception of exonic-circular RNAs (ecircRNAs), intronic-circular RNAs (icircRNAs), pregnancy. Although the evidence showed that circRNAs mediate various pathological processes in pregnancy, the mechanism of action is still unclear. In this paper, we provide a highlight of the current state of knowledge about circRNAs, its relation to preeclampsia, and the potential applications as preeclampsia biomarkers. and exon-intronic RNAs (EIcircRNAs). Spliceosome cuts the exonic and intronic sequences. The remaining intrusions are finally connected to form complete circRNA (Figure 1). ${ }^{6}$ The circRNAs can be transported by extracellular vesicles (EV). The circRNA is also removed from cells into the extracellular space via the $\mathrm{EV}$, and the released EV can be taken up by other cells, which shows that circRNA can somehow play a role in cellular communication. $^{7}$

Aninterestingfindingshowed thatconcentrations of EVs in maternal plasma increase along with the development of pregnancy. Pregnancy-related EV contains protein, mRNAs, miRNAs, and circRNAs with various biological functions. ${ }^{8}$ Uncovering the interaction of various cells in the mother and fetal interface through EV-containing circRNA can help us extend our understanding of the molecular pathology in complicated pregnancy.

\section{CIRCRNA FUNCTION}

The biological activity of circRNAs has been slowly uncovered. However, when compared with miRNAs and other ncRNAs, knowledge about the function of circRNAs is by far very limited. The potential function of circRNAs includes roles as a miRNAs sponge or as competing-endogenous RNAs (ceRNAs), interacts with RNA-binding proteins (RBP), and regulates the transcription of genes and the translation of mRNAs (Figure 2). EcircRNAs 

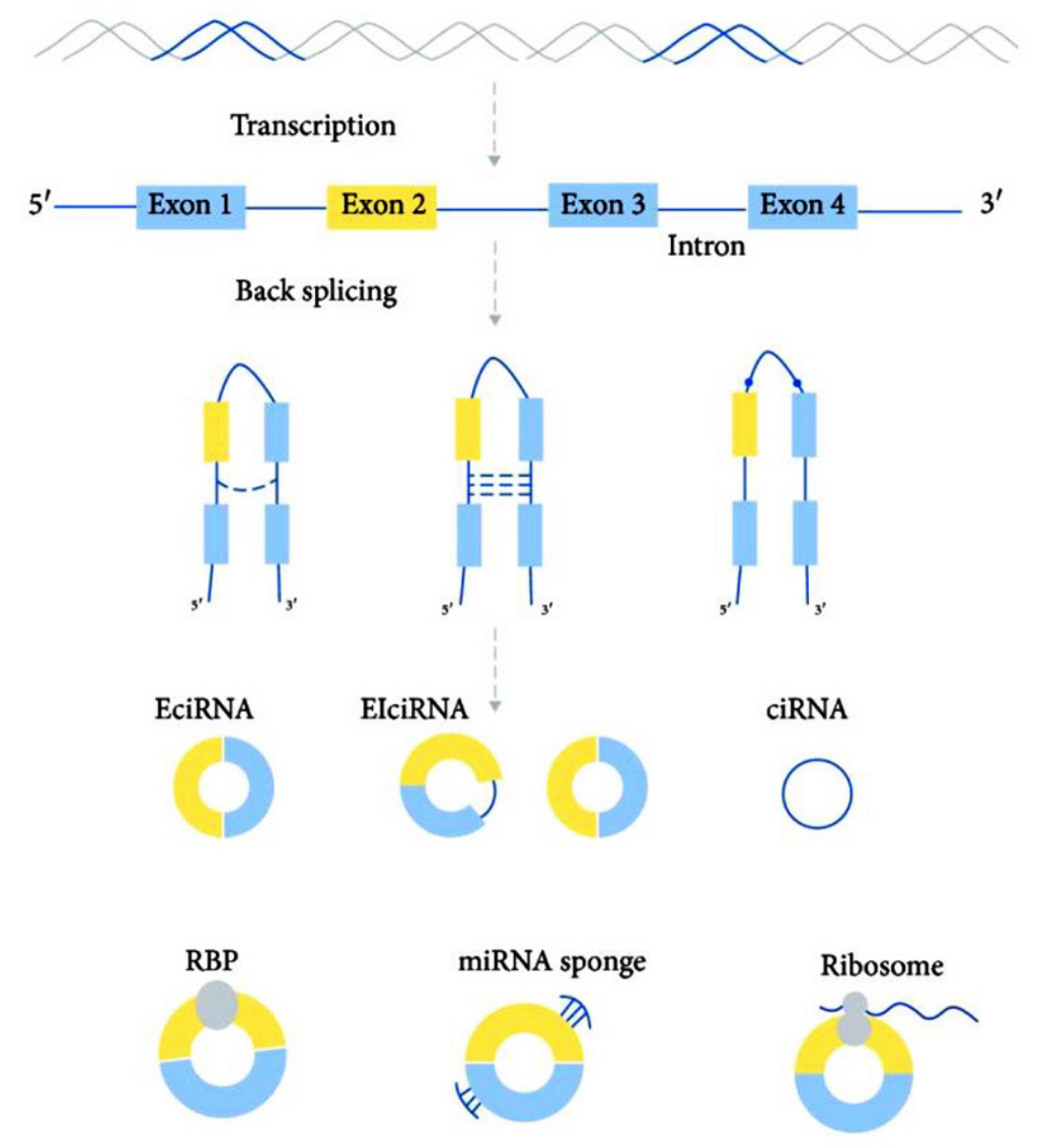

Figure 1. The schematic diagram of circRNAs formation and action. ${ }^{9}$

can act as a "miRNAs sponge" by storing miRNAs response elements (MREs) which regulate gene transcription and post-transcription processing. ${ }^{4}$ Normally when a miRNA assembled into the RNAinduced silencing complex (RISC), it finds the complementary target mRNAs and initiates the inhibition of translation or trigger the degradation of the mRNAs. The circRNAs have several MREs that allow them to compete with most miRNAs targets, which results in a reduce the inhibitory effect of miRNAs on the expression of target genes. Therefore, the circRNAs contribute to regulating the expression of genes at the transcriptional level..$^{10,11}$

In addition to the inhibitory activity against miRNAs, circRNAs also regulate gene expression and protein translation through interactions with molecules that involved in RNA editing and alternative splicing including RBP, such as RNA polymerase II (RNA Pol II), Argonaute protein (AGO), muscleblind protein (MBL), and vibration I (QKI). ${ }^{12,13}$ The circRNAs can tie up a single or multiple RBP and forming a stable, large complex of proteins. Interaction between circRNAs and RBP may produce effects that are similar to that previously described miRNAs sponge, thus leads to depletion of RBPs and subsequently reduce the interaction with the target RNAs. ${ }^{14}$ These findings illustrate that the circRNAs able to regulate the function and interactions of proteins. ${ }^{12,13}$

\section{THE ROLE OF THE CIRCRNAS IN PREECLAMPSIA}

Preeclampsia is a common disorder in pregnancy. Current estimation suggests that preeclampsia affects $3-8 \%$ of all pregnancy. Preeclampsia defined as pregnancy with blood pressure higher than 140/90 $\mathrm{mmHg}$ and proteinuria after 20 weeks of gestation. ${ }^{15,16}$ Although the pathophysiological mechanism of preeclampsia has not been fully elucidated, in general, it is agreed that dysregulation of the placenta plays an important role. Abnormalities of the placenta include abnormal invasion of the malformed vascular system, oxidative damage, and abnormal inflammatory response. ${ }^{17}$ Several studies have advocated that dysregulation of ncRNAs including circRNAs during the proliferation and invasion of trophoblast promote the pathogenesis of preeclampsia. ${ }^{17-19}$

Few landmark studies provide the early evidence of the involvement of circRNAs in preeclampsia. Zhou et al. showed that si-circ_3286 inhibits invasion of HTR8/Svneo cells. ${ }^{20}$ Other studies reveal that circRNA_0001855 and circRNA_0004904 affect the transcription of circulating pregnancyassociated plasma protein-A (PAPP-A) RNAs by competing with various types of miRNAs, resulting in its higher concentration in a pregnant woman who develops preeclampsia. ${ }^{21}$ Additionally, the circRNAs expression in the maternal-fetal interface and circulation of patients with preeclampsia compared to normal-healthy pregnancy were different (Figure 2). ${ }^{9}$

Although there are only handfuls of evidence point to a relationship of circRNAs and preeclampsia, the evidence of an association between miRNAs and preeclampsia has been extensive. Preeclampsia that requires termination of pregnancy at gestational age less than 34 weeks was associated with the downregulation of miR26a-5p. Some relevant circular RNAs has been shown to interact with miR-26a-5p, including circ_104823, circ_104824, and circ_104819.22 Other finding revealed the involvement of miR134. The miR-134 is significantly upregulated in preeclampsia and correlated negatively with the expression of ITGB1. The miR-134 suppress the infiltration of trophoblast cells by targeting ITGB1. 


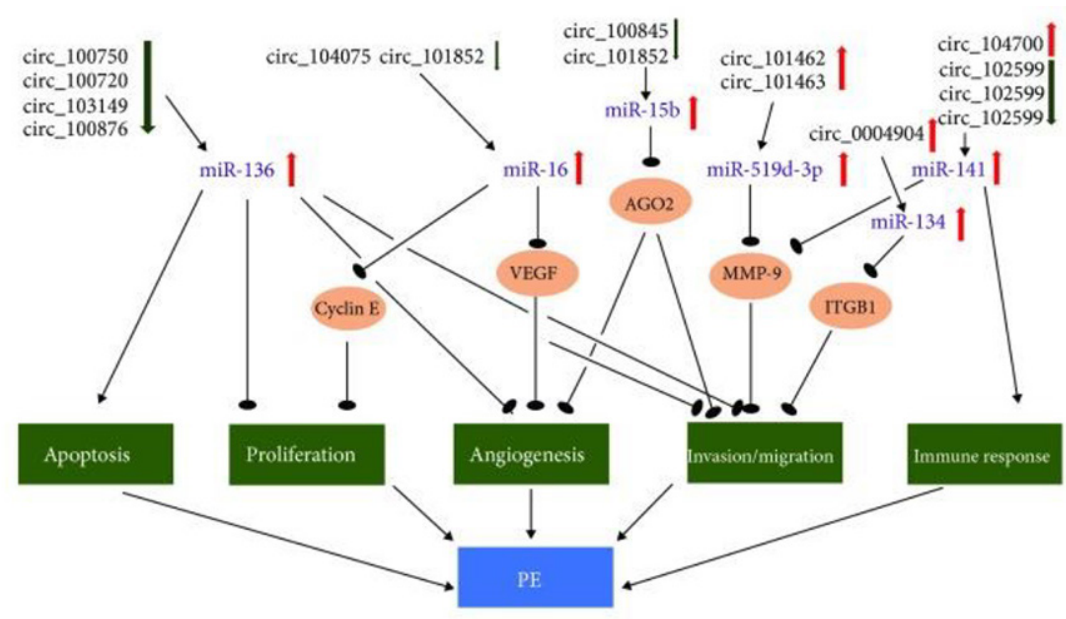

Figure 2. The network diagram of various circRNAs action as miRNAs sponge in the maternal-fetal interface that contributes to the pathogenesis of preeclampsia. ${ }^{9}$

At the same time, few circRNAs compete for a share of miR-134. ${ }^{21} \mathrm{Ji}$ et al. discovered that other miRNA, the miR-136 is significantly increased apoptosis, inhibits angiogenesis, and trophoblast invasion. ${ }^{23}$ The circRNAs which correlates with miR-16 and miR-136 were found to be down-regulated in preeclampsia. It is also suggested that miR-15b inhibited trophoblast cell invasion and endothelial cell tube formation through the downregulation of AGO2 expression. ${ }^{24}$ The miR-519d-3p expression is higher in the placenta of preeclampsia patients, and it may suppress the expression of MMP9, which affects the migration and invasion of trophoblast cells. ${ }^{25}$ Figure 2 showed the overall expression profile of circRNAs with the associated miRNAs. Our understanding of the molecular pathogenesis of preeclampsia is far from complete. However, currently available evidence conclude that abnormal expression of circRNAs associated with complications during pregnancy. ${ }^{21,26}$

\section{POTENTIAL APPLICATION}

Researchers suggest that circRNAs may play a role as a biological marker for preeclampsia. Compared to linear RNAs, circRNAs are more stable due to the unique structure of circRNAs that help avoid degradation. ${ }^{27}$ Besides that, circRNAs expression has specific-network and a specific-stage of development characteristics. ${ }^{28}$ Jiang et al. reported that the profile of circRNAs expression in patients with preeclampsia was different from a healthy pregnancy. Additionally, the study showed that circ_0004904, circ_0001855, and PAPP-A, when combined, could act as biomarkers for the detection of preeclampsia even before the onset of symptoms. ${ }^{21}$ Other studies have shown that circ_0036877 plays a role as ceRNA and shown potential as a biomarker for early-onset preeclampsia. ${ }^{26}$ Other studies also showed that circ_101222 is significantly higher in the blood cell of preeclamptic women. When combined with Endoglin plasma protein, it shows potential as a screening biomarker. ${ }^{29}$ These findings indicate that circRNAs may have a predictive value of preeclampsia. Moreover, through EV, circRNAs can be released to the extracellular space. Given that $\mathrm{EV}$ is present in body fluids, it makes a noninvasive diagnostic approach feasible.

Apart from its diagnostic capabilities, ncRNAs, including circRNAs, could be used as a treatment target. The decay of circRNAs from its primary cell into the circulation may be related to the communication between the cells throughout the body. Recently a novel method has been developed to eliminate ncRNAs in cancer, and similar principles could be used to target circRNAs. ${ }^{30,31}$ Targeting certain ncRNAs could affect multiple pathways, thus can cause side effects. Therefore, the function of certain circRNAs (or other ncRNA) must be fully characterized before it is applied as a target of treatment.

\section{CONCLUSION}

The role of circRNAs been slowly uncovered. Although our understanding of circRNAs role in regulating gene expression is still at an early stage, accumulated evidence showed that circRNAs has an important role in the various pathological process of pregnancy, including preeclampsia. On the other hand, the circRNAs have shown the characteristics that promote its uses as a diagnostic biomarker. In the future, it would be imperative to identify more circRNAs involvement in the various pathological process of pregnancy.

\section{AUTHOR CONTRIBUTION}

All authors have contributed substantially during concepting of the manuscript, gathering and analyzing related literature, drafting and revising the manuscript, giving final approval, and have agreeing to be accountable.

\section{CONFLICT OF INTEREST}

All authors declare there is no conflict of interest.

\section{FUNDING}

All authors have no external support or funding to report. 


\section{REFERENCE}

1. Wang Y, Mo Y, Gong Z, Yang X, Yang M, Zhang S, et al. Circular RNAs in human cancer. Mol Cancer. 2017;16(1). Available from: https://doi.org/10.1186/s12943-017-05987

2. Wang Q, Qu L, Chen X, Zhao Y-H, Luo Q. Progress in Understanding the Relationship Between Circular RNAs and Neurological Disorders. J Mol Neurosci. 2018;65(4):546-56. Available from: https://doi. org/10.1007/s12031-018-1125-Z

3. Gao L, Liu Y, Guo S, Yao R, Wu L, Xiao L, et al. Circulating Long Noncoding RNA HOTAIR is an Essential Mediator of Acute Myocardial Infarction. Cell Physiol Biochem. 2017;44(4):1497-508. Available from: http://www.ncbi. nlm.nih.gov/pubmed/29258067

4. Li Z, Huang C, Bao C, Chen L, Lin M, Wang X, et al. Exonintron circular RNAs regulate transcription in the nucleus. Nat Struct Mol Biol. 2015;22(3):256-64. Available from: https://doi.org/10.1038/Fnsmb.2959

5. Werfel S, Nothjunge S, Schwarzmayr T, Strom T-M, Meitinger T, Engelhardt S. Characterization of circular RNAs in human, mouse and rat hearts. J Mol Cell Cardiol. 2016;98:103-7. Available from: https://doi.org/10.1016/j. yjmcc.2016.07.007

6. Talhouarne GJS, Gall JG. Lariat intronic RNAs in the cytoplasm of Xenopus tropicalisoocytes. RNA. 2014;20(9):1476-87. Available from: https://doi. org/10.1261/rna.045781.114

7. Lasda E, Parker R. Circular RNAs Co-Precipitate with Extracellular Vesicles: A Possible Mechanism for circRNA Clearance. PLoS One. 2016;11(2):e0148407. Available from: https://doi.org/10.1371/journal.pone.0148407

8. Adam S, Elfeky O, Kinhal V, Dutta S, Lai A, Jayabalan N, et al. Review: Fetal-maternal communication via extracellular vesicles Implications for complications of pregnancies. Placenta. 2017;54:83-8. Available from: https://doi. org/10.1016/j.placenta.2016.12.001

9. Jia N, Li J. Role of Circular RNAs in Preeclampsia. Dis Markers. 2019;2019:7237495. Available from: http://www. ncbi.nlm.nih.gov/pubmed/31191755

10. Tay Y, Rinn J, Pandolfi PP. The multilayered complexity of ceRNA crosstalk and competition. Nature. 2014;505(7483):344-52. Available from: https://doi. org/10.1038/nature12986

11. Hansen TB, Jensen TI, Clausen BH, Bramsen JB, Finsen B, Damgaard CK, et al. Natural RNA circles function as efficient microRNA sponges. Nature. 2013;495(7441):3848. Available from: https://doi.org/10.1038/Fnature11993

12. Du WW, Zhang C, Yang W, Yong T, Awan FM, Yang BB. Identifying and Characterizing circRNA-Protein Interaction. Theranostics. 2017;7(17):4183-91. Available from: https://doi.org/10.7150/thno.21299

13. Conn S, Pillman K, Toubia J, Conn V, Salmanidis M, Phillips $\mathrm{C}$, et al. The RNA Binding Protein Quaking Regulates Formation of circRNAs. Cell. 2015;160(6):1125-34. Available from: https://doi.org/10.1016/j.cell.2015.02.014

14. van Rossum D, Verheijen BM, Pasterkamp RJ. Circular RNAs: Novel Regulators of Neuronal Development. Front Mol Neurosci. 2016;9(74). Available from: https://doi. org/10.3389/fnmol.2016.00074

15. Mol BWJ, Roberts CT, Thangaratinam S, Magee LA, de Groot CJM, Hofmeyr GJ. Pre-eclampsia. Lancet. 2016;387(10022):999-1011. Available from: https://doi. org/10.1016/s0140-6736\%2815\%2900070-7

16. Adnyana IBP, Liwang F, Negara KS, Manuaba IBP, Bhargah A, Prabawa IPY. Clinical risk factor of preeclampsia: a five-year retrospective study in Bali Royal Hospital, BaliIndonesia. gineco.eu. 2018;14(53):89-93. Available from: http://dx.doi.org/10.18643/gieu.2018.89

17. Song X, Luo X, Gao Q, Wang Y, Gao Q, Long W. Dysregulation of LncRNAs in Placenta and Pathogenesis of Preeclampsia. Curr Drug Targets. 2017;18(10). Available from: https://doi.org/10.2174/13894501186661704041600 00

18. Biró O, Nagy B, Rigó J. Identifying miRNA regulatory mechanisms in preeclampsia by systems biology approaches. Hypertens Pregnancy. 2016;36(1):90-9. Available from: https://doi.org/10.1080/10641955.2016.12 39736

19. Jiang J, Zhao Z-M. LncRNA HOXD-AS1 promotes preeclampsia progression via MAPK pathway. Eur Rev Med Pharmacol Sci. 2018;22(24):8561-8. Available from: http:// www.ncbi.nlm.nih.gov/pubmed/30575894

20. Zhou W, Wang H, Wu X, Long W, Zheng F, Kong J, et al. The profile analysis of circular RNAs in human placenta of preeclampsia. Exp Biol Med. 2018;243(14):1109-17. Available from: https://doi.org/10.1177/1535370218813525

21. Jiang $M$, Lash G, Zhao X, Long Y, Guo C, Yang $\mathrm{H}$. CircRNA-0004904, CircRNA-0001855, and PAPP-A: Potential Novel Biomarkers for the Prediction of Preeclampsia. Cell Physiol Biochem. 2018;46(6):2576-86. Available from: https://doi.org/10.1159/000489685

22. Hromadnikova I, Kotlabova K, Hympanova L, Krofta L. Cardiovascular and Cerebrovascular Disease Associated microRNAs Are Dysregulated in Placental Tissues Affected with Gestational Hypertension, Preeclampsia and Intrauterine Growth Restriction. Jeyaseelan K, editor. \{PLOS\} \{ONE\}. 2015;10(9):e0138383. Available from: https://doi.org/10.1371/journal.pone.0138383

23. Ji L, Zhang L, Li Y, Guo L, Cao N, Bai Z, et al. MiR-136 contributes to pre-eclampsia through its effects on apoptosis and angiogenesis of mesenchymal stem cells. Placenta. 2017;50:102-9. Available from: https://doi. org/10.1016/j.placenta.2017.01.102

24. Yang M, Chen Y, Chen L, Wang K, Pan T, Liu X, et al. miR15b-AGO2 play a critical role in HTR8/SVneo invasion and in a model of angiogenesis defects related to inflammation. Placenta. 2016;41:62-73. Available from: https://doi. org/10.1016/j.placenta.2016.03.007

25. Qian Y, Lu Y, Rui C, Qian Y, Cai M, Jia R. Potential Significance of Circular $\{$ RNA $\}$ in Human Placental Tissue for Patients with Preeclampsia. Cell Physiol Biochem. 2016;39(4):1380-90. Available from: https://doi. org/10.1159/000447842

26. Hu X, Ao J, Li X, Zhang H, Wu J, Cheng W. Competing endogenous RNA expression profiling in pre-eclampsia identifies hsa_circ_0036877 as a potential novel blood biomarker for early pre-eclampsia. Clin Epigenetics. 2018;10(1). Available from: https://doi.org/10.1186/ s13148-018-0482-3

27. Petkovic S, Müller S. RNA circularization strategies in vivo and in vitro. Nucleic Acids Res. 2015;43(4):2454-65. Available from: https://doi.org/10.1093/nar/gkv045

28. Jeck WR, Sorrentino JA, Wang K, Slevin MK, Burd CE, Liu J, et al. Circular RNAs are abundant, conserved, and associated with ALU repeats. RNA. 2012;19(2):141-57. Available from: https://doi.org/10.1261/Frna.035667.112

29. Zhang Y-G, Yang H-L, Long Y, Li W-L. Circular RNA in blood corpuscles combined with plasma protein factor for early prediction of pre-eclampsia. $\{B J O G\}$ An Int J Obstet Gynaecol. 2016;123(13):2113-8. Available from: https:// doi.org/10.1111/1471-0528.13897

30. Wang S, Hui Y, Li X, Jia Q. Silencing of lncRNA CCDC26 Restrains the Growth and Migration of Glioma Cells In Vitro and In Vivo via Targeting miR-203. Oncol Res Featur Preclin Clin Cancer Ther. 2018;26(8):1143-54. Available from: https://doi.org/10.3727/096504017x14965095236521 
31. Qian Y, Wang X, Ruan H, Rui C, Mao P, Cheng Q, et al. Circular RNAs expressed in chorionic villi are probably involved in the occurrence of recurrent spontaneous abortion. Biomed Pharmacother.2017;88:1154-62. Available from: https://doi.org/10.1016/j.biopha.2017.01.172

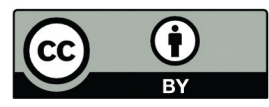

This work is licensed under a Creative Commons Attribution 